\title{
Influência do tratamento dentinário com antimicrobianos na resistência de união dos sistemas adesivos
}

\author{
Influence of dentinal treatment with antimicrobials in bond strength of \\ adhesive systems
Influencia del tratamiento dentinario con antimicrobianos en la resistencia de unión de los sistemas adhesivos

\author{
Eloiza Leonardo de MELO ${ }^{1}$ \\ Fernanda Suely Barros DANTAS ${ }^{2}$ \\ Leonardo José Rodrigues de OLIVEIRA ${ }^{3}$ \\ Rodivan BRAZ ${ }^{4}$ \\ Francineide Guimarães CARNEIRO ${ }^{5}$ \\ Olimpia Crispim da SILVEIRA
}
Mestranda em Odontologia, Área de concentração em Dentística/Endodontia, Universidade de Pernambuco, 54753-220, Camaragibe, PE, Brasil ${ }^{2}$ Graduada em Odontologia pela Universidade Estadual da Paraíba, 58429-500, Campina Grande, PE, Brasil
${ }^{3}$ Doutorando em Odontologia, Área de concentração em Dentística/Endodontia, Universidade de Pernambuco, 54753-220, Camaragibe, PE, Brasil ${ }^{4}$ Professor de Dentística da Universidade de Pernambuco, 54753-220, Camaragibe, PE, Brasil.
${ }^{5}$ Professora de Dentística da Universidade Estadual da Paraíba, 58429-500, Campina Grande, PE, Brasil

\begin{abstract}
Resumo
Introdução: A adesão é um fenômeno ligado à natureza do substrato e a composição dos sistemas adesivos, que, por sua vez sofrem constantes mudanças em sua composição. Neste contexto é relevante um estudo do comportamento dos sistemas adesivos sobre protocolos de desinfecção cavitária Objetivo: Avaliar a influência do tratamento de superfície dentinária com antimicrobianos na resistência de união de sistemas adesivos de condicionamento ácido total e autocondicionantes. Material e Método: Trinta dentes humanos incluídos em corpos de prova foram realizados os preparos cavitários e divididos em dois grupos de acordo com a estratégia de adesão utilizada: de condicionamento ácido total - Adper Single Bond 2 e Adper Scotchbond multi-uso e autocondicionates - Adper Single Bond 2 e Adper Scotchbond multi-uso. Estes por sua vez, tiveram seus subgrupos: controle, aplicação de clorexidina $2 \%$ e álcool 70 , que foram restaurados e submetidos à teste resistência de união. Resultados: A análise estatística foi realizada através de teste ANOVA e teste post hoc de Bonferroni, com intervalos de confiança de $99 \%$ nível de significância de $\mathrm{p} \leq 0,001$. Nos adesivos de condicionamento ácido total a utilização da clorexidina $2 \%$ e álcool 70 parece não alterar significativamente a resistência de união. Nos adesivos autocondicionantes o grupo controle apresentou divergências nos resultados entre os adesivos. Conclusão: para os adesivos universais a técnica de adesão úmida se mostrou mais indicada. Já para os adesivos de condicionamento ácido total a indicação de técnica varia de acordo com o adesivo.
\end{abstract}

Descritores: Adesivos Dentinários; Ligações de Hidrogênio; Reagentes para Ligações Cruzadas.

\section{Abstract}

Introduction: Adhesion is a phenomenon linked to the nature of the substrate and the composition of adhesive systems, which in turn undergoes constant changes in its composition. In this context it is relevant to study the behavior of adhesive systems on cavity disinfection protocols Aim: To evaluate the influence of dentin surface treatment with antimicrobials on the bond strength of adhesive systems of total acid etching and self - etching. Methods: Thirty human teeth included in test specimens were submitted to cavity preparation and divided into two groups according to the adhesion strategy: total acid conditioning - Adper Single Bond 2 and Adper Scotchbond multi-use and autocondicionates - Adper Single Bond 2 and Adper Scotchbond multi-use. These, in turn, had their subgroups: control, application of chlorhexidine $2 \%$ and alcohol 70 , which were restored and submitted to the bond strength test. Results: Statistical analysis was performed using ANOVA and post hoc Bonferroni test, with confidence intervals of $99 \%$ significance level of $p \leq 0.001$. In total acid conditioning adhesives the use of $2 \%$ chlorhexidine and alcohol 70 does not appear to significantly change the bond strength. In the self-etching adhesives the control group showed differences in the results between the adhesives. Conclusion: for the universal adhesives the wet adhesion technique was more indicated. For the total acid conditioning adhesives, the technique indication varies according to the adhesive

Descriptors: Dentin-Bonding Agents; Hydrogen Bonding; Cross-Linking Reagents.

\section{Resumen}

Introducción: La adhesión es un fenómeno ligado a la naturaleza del sustrato y la composición de los sistemas adhesivos, que a su vez sufren constantes cambios en su composición. En este contexto es relevante un estudio del comportamiento de los sistemas adhesivos sobre protocolos de desinfección cavitaria. Objetivo: Evaluar la influencia del tratamiento de superficie dentinaria con antimicrobianos en la resistencia de unión de sistemas adhesivos de acondicionamiento ácido total y autocondicionantes. Material y Método: Treinta dientes humanos incluidos en cuerpos de prueba se realizaron los preparativos cavitarios y divididos en dos grupos de acuerdo con la estrategia de adhesión utilizada: de acondicionamiento ácido total - Adper Single Bond 2 y Adper Scotchbond multiuso y autocondicionates - Adper Single Bond 2 y Adper Scotchbond multiuso. Estos a su vez, tuvieron sus subgrupos: control, aplicación de clorexidina 2\% y alcohol 70, que fueron restaurados y sometidos a la prueba resistencia de unión. Resultados: El análisis estadístico fue realizado a través de test ANOVA y prueba post hoc de Bonferroni, con intervalos de confianza del 99\% nivel de significancia de p $\leq 0,001$. En los adhesivos de acondicionamiento ácido total la utilización de la clorexidina $2 \%$ y el alcohol 70 parece no alterar significativamente la resistencia de unión. En los adhesivos autocondicionantes el grupo control presentó divergencias en los resultados entre los adhesivos. Conclusión: para los adhesivos universales la técnica de adhesión húmeda se mostró más indicada. Para los adhesivos de acondicionamiento ácido total la indicación de técnica varía de acuerdo con el adhesivo.

Descriptores: Recubrimientos Dentinarios; Enlaces de Hidrógeno; Reactivos de Enlaces Cruzados.

\section{INTRODUÇÃO}

A adesão aos tecidos dentários trouxe um avanço considerável para a Odontologia, porém, um protocolo adesivo que elimine a sensibilidade pós-operatória e proporcione longevidade ao procedimento restaurador ainda é questionável na literatura atual ${ }^{1}$. Fatores fisiológicos e patológicos da dentina, como profundidade cavitária, idade do paciente, composição orgânica e aquosa podem interferir na adesão a este substrato. Protocolos clínicos de preparo da superfície dentinária têm objetivado aumentar a longevidade e reduzir a microinfiltração e nanoinfiltração, que degradam a interface adesiva $^{2}$.

Os sistemas adesivos, atualmente são classificados de acordo com o tipo de tratamento com a smear layer: os de "condicionamento ácido total" (total etch) que removem a smear layer e "autocondicionantes" (selfetch) ou de passo único, onde condicionam e hibridizam o substrato, incluindo a smear layer na formação da camada hibrida ${ }^{3}$. Entretanto, a presença de água residual na matriz colágena desmineralizada 
pode provocar a separação de fase dos adesivos, dificultar a completa evaporação dos solventes, e promover uma polimerização incompleta dos monômeros, em torno das fibrilas colágenas, gerando porosidades na camada híbrida e reduzindo suas propriedades mecânicas ${ }^{4}$.

Pré-tratamentos como condicionamento ácido (seletivo ou não), abrasão com glicina, lavagem com clorexidina ou etanol são comumente utilizados para aumentar a resistência de união e reduzir o conteúdo bacteriano da cavidade ${ }^{14}$. A "técnica úmida"consiste em substituir lentamente, a água presente na matriz dentinária ${ }^{5}$. Isso é feito com lavagens sequenciais de etanol em concentrações ascendentes, para impedir o colapso dos espaços interfibrilares da matriz de colágeno e permitir a infiltração de monômeros hidrófobos. O objetivo seria formar uma camada híbrida mais durável e menos propensa à sorção de água. $\mathrm{O}$ emprego da técnica úmida na dentina é justificado pela manutenção dos espaços interfibrilares para a infiltração dos monômeros. Entretanto, não apenas a água é capaz de manter as fibrilas colágenas expandidas, mas também alguns solventes anidros, como o etanol ${ }^{6}$.

Estudos demonstraram que o etanol pode ser utilizado como um solvente para monômeros e, também, como um agente que promova a substituição da água na dentina desmineralizada para procedimentos adesivos ${ }^{7}$. Como o etanol é um melhor solvente para os monômeros, qualquer resíduo de etanol deixado nas fibrilas colágenas permitirá a dissolução dos monômeros, e um melhor envelopamento das fibrilas colágenas pelo adesivo, ao contrário do que acontece com a água ${ }^{3,8}$. A matriz dentinária desmineralizada, saturada com etanol, apresenta uma diminuição do diâmetro das fibrilas de colágeno, criando assim, espaços interfibrilares mais amplos, promovendo uma maior infiltração de resinas e um aumento na resistência de união $0^{9,10}$.

A clorexidina $2 \%$ é outra substância comumente utilizada para desinfecção de preparos cavitários, entretato, além da capacidade antimicrobiana sabe-se que a clorexidina $2 \%$ atua na inibição das metaloproteínases (MMPs), responsáveis pela degradação e remodelação do colágeno existente na dentina, um fator relevante para ser considerado, visto que as fibras colágenas são essenciais no processo de formação da camada híbrida e ancoragem das restaurações adesivas $^{2,16}$

Considerando a importância de estudos que abordem a influência do tratamento dentinário com antimicrobianos e solventes na resistência de união dos sistemas adesivos, o presente estudo se propôs a avaliar a influência do emprego da clorexidina a $2 \%$ e do álcool a $70 \%$ sobre a resistência de união em dentina, por microcisalhamento, comparando diferentes sistemas adesivos convencionais e autocondicionantes.

\section{MATERIAL E MÉTODO}

Foram incluídos no estudo quatro sistemas adesivos: Adper Single Bond 2 (ASB2), Single Bond Universal (SBU), Adper Scotchbond multi-uso (ASMU) e Clearfil SE BOND (CSB), sendo dois de condicionamento total (ASB2 e ASMU) e dois autocondicionantes (SBU e CSB) (Tabela 1).

A resina composta selecionada para o estudo foi a nanoparticulada (Filtek Z350 XT ${ }^{\circledR}$ A1, 3M ESPE, St. Paul, MN, EUA).

\section{- Confecção dos Corpos de Prova}

Trinta dentes humanos, foram incluídos em cilindros plásticos de PVC com $20 \mathrm{~mm}$ de diâmetro e $20 \mathrm{~mm}$ de altura, com a face vestibular do dente voltada para cima e projetada $1 \mathrm{~mm}$ além da borda do tubo plástico de PVC, em seguida os espaços vazios foram preenchidos com resina acrílica.

O desgaste da superfície dentária para remoção da tampa oclusal foi realizado sob refrigeração, com o auxílio de politriz (ALP-4) e lixas de granulações (180, 320, 400 e 600).
Ao final os espécimes foram armazenados em água destilada a temperatura ambiente.

As trinta amostras foram divididas em 04 grupos: Grupo I Single Bond Universal (3M/ESPE), Grupo II Adper Single Bond 2 (3M/ESPE), Grupo III Adper Scotchbond multi-uso (3M/ESPE) e Grupo IV Clearfil SE BOND (KURARAY) (Tabela 2).

Cada grupo foi dividido em três subgrupos: I-Controle (sem aplicação de qualquer substância na superfície), IIDesinfecção com Clorexidina $2 \%$ e III-Desinfecção com álcool 70\% (Tabela 2).

Tabela 1. Sistemas adesivos e sua composição

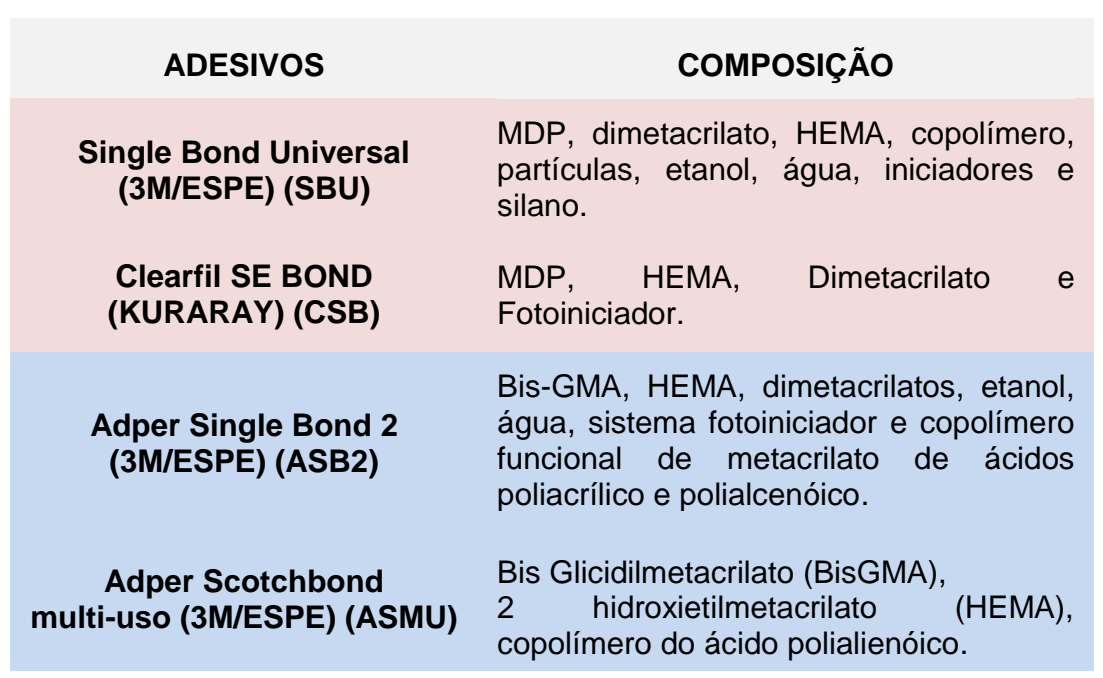

Tabela2. Grupos e Subgrupos

\begin{tabular}{|c|c|c|c|c|}
\hline & \multicolumn{4}{|c|}{ GRUPOS DE ADESIVOS } \\
\hline & \multicolumn{2}{|c|}{$\begin{array}{l}\text { CONDICIONAMENTO } \\
\text { ÁCIDO TOTAL }\end{array}$} & \multicolumn{2}{|c|}{ AUTOCONDICIONANTES } \\
\hline & ASB2 & ASMU & SBU & CSB \\
\hline \multirow{3}{*}{ 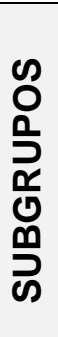 } & Controle & Controle & Controle & Controle \\
\hline & $\begin{array}{c}\text { Aplicação } \\
\text { de } \\
\text { Clorexidina } \\
2 \%\end{array}$ & $\begin{array}{c}\text { Aplicação } \\
\text { de } \\
\text { Clorexidina } \\
2 \%\end{array}$ & $\begin{array}{c}\text { Aplicação de } \\
\text { Clorexidina } \\
2 \%\end{array}$ & $\begin{array}{l}\text { Aplicação de } \\
\text { Clorexidina } 2 \%\end{array}$ \\
\hline & $\begin{array}{l}\text { Aplicação } \\
\text { de álcool } \\
70 \%\end{array}$ & $\begin{array}{c}\text { Aplicação } \\
\text { de álcool } \\
70 \%\end{array}$ & $\begin{array}{l}\text { Aplicação de } \\
\text { álcool } 70 \%\end{array}$ & $\begin{array}{c}\text { Aplicação de álcool } \\
70 \%\end{array}$ \\
\hline
\end{tabular}

\section{- Preparação das Superfícies}

As amostras dos grupos do ASB2 e ASMU foram condicionadas com ácido fosfórico $37 \%$, por 15 segundos em dentina e logo após, cada subgrupo recebeu seus respectivos tratamentos: Controle, aplicação de álcool 70\%, e aplicação de clorexidina $2 \%$. As soluções foram aplicadas de forma ativa com o microbush por 60 segundos, seguida da lavagem por 30 segundos e remoção do excesso de umidade com papel feltro hidrófilo.

Para as amostras dos grupos SBU e CSB foram realizados apenas os tratamentos controle, aplicação de álcool $70 \%$ e aplicação de clorexidina $2 \%$.

Em todos os grupos, os sistemas adesivos foram aplicados em duas camadas com auxílio do microbush e fotoativados com aparelho Radii-cal (SDI), potência de $1.200 \mathrm{nw} / \mathrm{cm}^{2}$ por 20 segundos.

A resina composta nanoparticulada $\mathrm{Z} 350$ XP (3M Espe) foi inserida na matriz de tygon (dispositivo utilizado para confecção de cilindros de resina composta), sendo posteiormente fotopolimerizada por 60 segundos. Concluída a confecção dos cilindros de resina composta sobre os espécimes, as matrizes de tygon foram removidas, obtendo-se os corpos de prova, que foram armazenados em estufa à $37^{\circ} \mathrm{C}$ e que após 24 horas, foram submetidos a teste de microcisalhamento em máquina de ensaios universais (INSTRON 5582).

\section{- Análise Estatística}

Os valores obtidos do microcisalhamento foram analisados no Programa Estatístico SPSS (Versão 17), através 
de teste ANOVA com as variáveis independentes (grupos experimentais e adesivos) e com a variável dependente a resistência de união em Mpa, utilizou-se então o teste post hoc de Bonferroni. Considerando o tamanho da amostra, optou-se por utilizar intervalos de confiança de $99 \%$ e uma significância de $\mathrm{p} \leq 0,001$.

\section{RESULTADOS}

As médias de resistência de união por microtração (MPa) e os desvios padrões são apresentados na Tabela 3.

Tabela 3. Média e desvio padrão dos subgrupos em relação aos adesivos

\begin{tabular}{ccc}
\hline SUBGRUPOS & ADESIVOS & MÉDIA \pm DP \\
\hline \multirow{3}{*}{ Controle - SG-I } & ASB2 & $15,6 \pm 11,3$ \\
& CSB & $17,7 \pm 12,7$ \\
& ASMU & $41,1 \pm 39,1$ \\
& SBU & $47,8 \pm 34,9$ \\
\hline \multirow{2}{*}{ Álcool a 70\%- SG-II } & ASB2 & $35,1 \pm 22,1$ \\
& CSB & $9,9 \pm 5,3$ \\
& ASMU & $37,4 \pm 29,8$ \\
& SBU & $49,0 \pm 33,9$ \\
\hline & ASB2 & $34,0 \pm 34,1$ \\
Clorexidina a 2\%- SG-III & CSB & $29,9 \pm 25,4$ \\
& ASMU & $63,3 \pm 59,6$ \\
& SBU & $35,5 \pm 29,5$ \\
\hline
\end{tabular}

\section{DISCUSSÃO}

Nos subgrupos que utilizaram a clorexidina a $2 \%$ observou-se que o ASMU apresentou os maiores valores de resistência de união, comparado ao SBU. O emprego desta solução, previamente à aplicação dos sistemas adesivos retarda a degradação das interfaces adesivas aumentando a longevidade da camada híbrida ${ }^{11,12}$. Devido ao ASMU utilizar estratégia adesiva de condicionamento ácido total há uma maior estimulação da matriz de MMPs devido a grande acidez gerada pelo ácido fosfórico em contato com a dentina $^{15}$. Assim sendo, parece ser extremamente importante utilizar-se neste tipo de sistema adesivo (convencionais) clorexidina $2 \%$ como demonstrado nos resultados do presente estudo.

Entretanto, em sistemas adesivos universais como o SBU os resultados mostraram um pequeno aumento na resistência de união quando se utilizou a clorexidina $2 \%$ e uma diminuição quando se utilizou álcool a 70\%. Podendo ainda num futuro breve ser utilizadas substancias que fortalecam as ligações cruzadas das fibras colágenas, chamadas substancias crosslinquings, reduzindo o poder da ação proteolítica das matrizes de MMPs, diminuindo assim a nanoinfiltração ${ }^{2}$.

O subgrupo controle apresentou resistência de união superior com o adesivo SBU, já o pior desempenho foi com o ASB2. O 10-MDP (methacryloxydecyl dihydrogen phosphate) é um monômero funcional acídico capaz de desmineralizar o tecido dentário, com um grupo polimerizável que interage com outros monômeros, e um grupo fosfatado capaz de se ligar à moléculas de cálcio dos tecidos dentários, esta ligação eletrostática do 10-MDP com a hidroxiapatita é determinante na resistência de união ${ }^{13}$. No seu estudo Wang et al. ${ }^{13}$ testou quatro adesivos (Clearfil SE Bond, Scotchbond Universal, Optibond XTR e Adper Easy One) sendo os dois primeiros com 10-MDP em sua composição, onde foram aplicados na dentina de acordo com as instruções do fabricante e foram submetidos à análise de microtração. $\mathrm{Na}$ análise com o Microscópio Eletrônico de Varredura ficaram evidenciadas ligações entre os monômeros e a dentina. Neste estudo a afirmativa de Wang et al. ${ }^{13}$ foi parcialmente confirmada, pois o sistema adesivo SBU apresentou um maior valor de resistência de união, entretanto o CSB, que também tem na sua composição o 10-MDP apresentou resultados baixos quando foi utilizado como subgrupo controle.

No subgrupo com tratamento com álcool $70 \%$ o SBU apresentou os maiores valores de resistência de união seguido do ASMU. Como o SBU e o ASMU possuem em sua composição o álcool etílico e outras substancias tidas como solventes polares, tal resultado pode ser explicado através do estudo de Pashley et al. ${ }^{7}$ que concluiu que as propriedades de tração da dentina são dependentes da capacidade de ligação do hidrogênio dos solventes polares, corroborando com o estudo em questão. Ademais, de acordo com Sauro et al. ${ }^{3}$, como o etanol é o melhor solvente para os monômeros, qualquer resíduo de etanol deixado nas fibrilas colágenas permitirá a dissolução dos monômeros, e um melhor envelopamento das fibrilas colágenas pelo adesivo, ao contrário do que acontece com a água.

\section{CONCLUSÃO}

Mesmo com as limitações deste estudo, evidenciou-se que o tratamento de superfície com a clorexidina $2 \%$ nos adesivos de condicionamento ácido total não afeta de maneira relevante a resistência de união, podendo ser utilizada como solução desinfetante.

No grupo controle o adesivo universal apresentou melhores resultados, reafirmando a importância do 10-MDP na composição dos adesivos e sua interação química com o subtrato dentinário.

A utilização do álcool não afeta de maneira considerável a resistência de união de todos os grupos adesivos.

\section{REFERÊNCIAS}

1. Santos de RA, Lima EA, Montes MAJR, Braz R. Pretreating dentin with chlorhexadine and CPP-ACP: selfetching and universal adhesive systems. Acta Biomater Odontol Scand. 2016; 2(1):79-85.

2. Zhang Z, Mutluayb M, Tezvergil-Mutluayb A,Tay FR, Pashley DH, Arola D. Effects of EDC crosslinking on the stiffness of dentin hybrid layers evaluated by nanoDMA over time. Dent Mater. 2017; 33(8):904-14.

3. Sauro S, Watson TF, Mannocci F, Tay FR, Pashley DH. Prevention of water contamination of ethanol-saturated dentin and hydrophobic hybrid layers. J Adhes Dent. 2009; 11(4):271-8.

4. Gupta A, Tavane P, Gupta PK, Tejolatha B, Lakhani AA, Tiwari $\mathrm{R}$ et al. Evaluation of microleakage with total etch, self etch and universal adhesive systems in class $\mathrm{V}$ restorations: an in vitro study. J Clin Diagn Res. 2017; 11(4):ZC53-ZC6.

5. Hosaka K, Nakajima M, Monticelli F, Carrilho M, Yamauti $\mathrm{M}$, Aksornmuang $\mathrm{J}$ et al. Influence of hydrostatic pulpal pressure on the microtensile bond strength of all-in-one self-etching adhesives. J Adhes Dent. 2007; 9(5):437-42.

6. Poggio C, Beltrami R, Colombo M, Chiesa M, Scribante A. Influence of dentin pretreatment on bond strength of universal adhesives. Acta Biomater Odontol Scand. 2017; 3(1):30-5.

7. Pashley DH, Agee KA, Carvalho RM, Lee KW, Tay FR, Callison TE. Effects of water and water-free polar solvents on the tensile properties of demineralized dentin. 2003; 19(5):347-52.

8. Eddleston CL, Hindle AR, Agee KA, Carvalho RM, Tay FR, Rueggeberg FA et al. Dimensional changes in aciddemineralized dentin matrices following the use of HEMA-water versus HEMA-alcohol primers. J Biomed Mater Res A. 2003; 67(3):900-7.

9. Kanka, J. Effect of resin primer solvents and surface 
wetness on resin composite bond strength to dentin. Am J Dent. 1992;5 (4):213-5.

10. Tay FR, Pashley DH, Kapur RR, Carrilho MR, Hur YB, Garrett LV et al. Bonding BisGMA to dentin a proof of concept for hydrophobic dentin bonding. J Dent Res. 2007; 86(11):1034-9.

11. Campos, EA, Correr GM, Leonardi DP, Barato-Filho F, Gonzaga CC, Zielak, JC. Chlorhexidine diminishes the loss of bond strength over time under simulated pulpal pressure and thermo-mechanical stressing. J Dent. 2009; 37(2):108-14.

12. Komori PC, Pashley DH, Tjäderhane L, Breschi L, Mazzoni A, de Goes MF et al. Effect of 2\% chlorhexidine digluconate on the bond strength to normal versus cariesaffected dentin. Oper Dent. 2009; 34(2):157-65.

13. Wang R, Shi Y, Li T, Pan Y, Cui Y, Xia W. Adhesive interfacial characteristics and the related bonding performance of four self-etching adhesives with different functional monomers applied to dentin. J Dent. 2017; 62:72-80.

14. Delvan GS. Sistemas Adesivos Dentinários [monografia]. Florianopolis: Escola de Aperfeiçoamento Professional, Curso de Especialização em Dentística Restauradora; 2001.

15. Gendron R, Grenier D, Sorsa T, Mayrand D. Inhibition of the activities of matrix metalloproteinases 2,8 , and 9 by chlorhexidine. Clin Diagn Lab Immunol. 1999; 6(3):437-9.

16. De Castro FL, De Andrade MF, Duarte Junior SL, Vaz LG, Ahid FJ. Effect of $2 \%$ chlorhexidine on microtensile bond strength of composite to dentin. J Adhes Dent. 2003; 5(2):129-38.

\section{CONFLITO DE INTERESSES}

Os autores declaram não haver conflitos de interesse.

\section{AUTOR PARA CORRESPONDÊNCIA}

Eloiza Leonardo de Melo

eloizaleonardo@hotmail.com

Submetido em 05/01/2018

Aceito em 15/02/2018 